\title{
Brane universe and holography in the spacetime of a charged AdS dilaton black hole
}

\author{
Ai-chen $\mathrm{Li} \odot$ \%* \\ Theoretical Physics Division, College of Applied Sciences, Beijing University of Technology, Beijing, China \\ and Departamento de matematica da Universidade de Aveiro and CIDMA, \\ Campus de Santiago, 3810-183 Aveiro, Portugal
}

(Received 13 January 2020; accepted 17 March 2020; published 17 April 2020)

\begin{abstract}
In the background of a charged anti-de Sitter (AdS) dilaton black hole, we investigate the movement of a self-graviting three-brane and relevant holographic effects as the brane moves close to the AdS boundary. The induced metric on the brane corresponds to an exact Friedmann-Lemaitre-Robertson-Walker (FLRW) geometry, while the evolution of brane is determined by the Israel junction condition and the effective Einstein field equation on the brane together. When the brane approaches the AdS boundary, AdS/CFT correspondence implies that a radiation dominated FLRW universe $\left(P=\frac{1}{3} \rho\right)$ should be given. According to the holographic renormalization procedure, we involve an appropriate surface counterterm into the gravitational action for achieving $P=\frac{1}{3} \rho$ on the brane. This surface counterterm also plays an important role in calculating the mass of a charged AdS dilaton black hole. Finally, we obtain the thermodynamic quantities and give an extended Cardy-Verlinde formula on the brane.
\end{abstract}

DOI: $10.1103 /$ PhysRevD.101.086019

\section{INTRODUCTION}

The study about physics beyond four-dimensional (4D) spacetime has made great progress in recent decades; a lot of interesting physics was obtained from either the phenomenological aspects or theoretical implications, such as $\mathrm{M}$ theory [1,2], anti de-Sitter space/conformal field theory $[3,4]$ (i.e., AdS/CFT) correspondence, and the brane world scenario in which our universe is viewed as a $3+1$ dimensional brane embedded in a higher-dimensional spacetime [5-7]. Actually, the original concept of brane could be traced back to the work of Rubakov and Shaposhnikov [8,9]; it was also developed to show that the fundamental gravitational scale could be close to Tev scale by introducing large extra spatial dimension transverse to the brane [10-12]. Compared to the previous ones, an important variant of brane world scenario involves warped compactification; this idea was first considered by RS-I model [6] in order to solve the gauge hierarchy problem, which introduced a five-dimensional antide Sitter (AdS) compactified extra dimension between two Minkowski branes with an exponential warp factor in metric solution. For implementing localization of 4D

\footnotetext{
*lac@emails.bjut.edu.cn, aichenli@visit.uaveiro.eu
}

Published by the American Physical Society under the terms of the Creative Commons Attribution 4.0 International license. Further distribution of this work must maintain attribution to the author(s) and the published article's title, journal citation, and DOI. Funded by SCOAP ${ }^{3}$. graviton and reproducing Newton's law on the visible brane, RS-II model was proposed, which has a noncompactified extra dimension. From perspective of phenomenology, unlike the Arkani-Dimopoulos-Dvali model [10-12], these warped models have less striking signature at colliders or in astrophysical processes and concomitantly being less constrained. In cosmological applications of brane world, RS-II model has been the most successful model since it provided the capability of modifying the structure of Einstein's field equations [13,14].

For applying brane world to cosmology, a timedependent solution needs to be constructed. There exist two physical scenarios, one focuses on finding timedependent bulk geometry as cosmological solution [15-18], and another one considers an alternative case in which the bulk remains to be static but the brane acquires a velocity, while the observers on the brane feel cosmological expansion or contraction as the brane moves [19-21]. Actually, some enriched physical phenomena will be uncovered when considering the movement of brane in AdS spacetime. More interesting, if one considers the AdS black hole as bulk spacetime for a motional brane, the physics of black hole, brane universe, and holography could be connected together naturally in this way. Under the situation of a motional brane in spacetime of AdS-Schwarzschild black hole, associating with the AdS/CFT correspondence and black hole thermodynamics, [22] generalize the Cardy formula [23] to the arbitrary-dimensional spacetime. Inspired by this work, the generalized Cardy formula (called by Cardy-Verlinde formula) has been checked in various black holes bulk with 
AdS asymptotics [24-39]. Besides, [40] constructs a holographic reheating model by considering the movement of a probe brane in AdS-Vaidya spacetime and the formation process of AdS black hole dual to the thermalization process of brane universe. Recently, by constructing a model of moving the brane in AdS-Schwarzschild black hole, the holographic complexity growth in an Friedmann-LemaitreRobertson-Walker (FLRW) universe is considered by [41].

As the low-energy effective theory of supergravity and string [42], dilaton gravitation theories have attracted many attentions in recent decades. Inspired by AdS/CFT, many works devote to finding the asymptotically AdS black hole solution in dilaton gravity with various self-interacting potential of dilaton. Particularly, it is of great interest to consider Liouville-type dilaton potential which originated from the supersymmetry breaking of a higher-dimensional supergravity model [43-45]. And [45,46] have proved that in models of one and two Liouville-type potential, there does not exist the asymptotically flat or asymptotically AdS black hole solution. By combining three different Liouville-type dilaton potentials, [47-49] obtain the asymptotically AdS black hole solution in EinsteinMaxwell-Dilaton theory. Based on this black hole solution, many interesting physical phenomenologies have been explored, like the black hole thermodynamics [50,51], holographic thermalization [52], black hole phase transition in extend phase space [53], and domain wall cosmology [54].

In this paper, our purpose is to consider the movement of brane in the charged AdS dilaton black hole solved by $[48,49]$ and the relevant holographic effects when the brane approaches the AdS boundary. As showed in [50,53], a rich phase structure of black hole thermodynamics could be uncovered when adjusting the dilaton coupling constant. Meanwhile, when considering the motion of the domain wall in this charged AdS dilaton black hole, [54] also observes enriched evolution modes of the domain wall universe as varying the value of the dilaton coupling constant. Hence, it seems that there exists a connection between the thermodynamics of the AdS dilaton black hole in the bulk spacetime and the evolution of FLRW universe on the brane/wall. Based on the idea proposed in [22], namely associating the black hole thermodynamics with AdS/CFT correspondence, [25] obtains a modified CardyVerlinde formula for a CFT living in the boundary of AdSRN black hole spacetime. In terms of the AdS dilaton black hole $[48,49]$, when we make the dilaton coupling constant $\alpha=0$, this solution will reduce to the AdS-RN one accordingly. Thus, when the brane approaches the AdS boundary, we naturally expect to give a similar CardyVerlinde formula on the brane like the one obtained by [25] but with some corrections of dilaton coupling constant $\alpha$. Note that the matter field confined on the brane is a general quantum field theory without conformal symmetry [55-57]. And we need to involve an appropriate surface counterterm to restore the conformal symmetry when the brane approaches the boundary of the AdS dilaton black hole. Although the authors [54] have considered the movement of the brane/wall in the AdS dilaton black hole $[48,49]$, they ignore the self-gravitating effects of the brane/wall. Thus, in their case, the evolution of brane/wall is controlled by the Israel junction condition [58] only. However, in our scenario, we involve the gravity on the brane by using the method provided in [13], and the evolution of brane is determined by the effective Einstein field equation on the brane and the Israel junction condition together.

Our work is organized as follows. In Sec. II, we briefly review the five-dimensional asymptotically AdS black hole solution and relevant thermodynamics. Also, we give a well-defined boundary stress-energy tensor and calculate the black hole mass by adding an appropriate surface counterterms to the gravitational action. The movement of self-gravitating brane in the background of the AdS dilaton black hole is considered in Sec. III. In Sec. IV, the holographic effects on the brane will be investigated as the brane approaches the AdS boundary. Finally, Sec. V will summarize our results and give a discussion.

\section{ADS DILATON BLACK HOLES AND RELEVANT THERMODYNAMICAL QUANTITIES}

\section{A. Dilaton black holes solution in asymptotically AdS spacetime}

An asymptotically AdS dilatonic black hole with charge will be considered as a background spacetime (i.e., bulk) for the brane's motion; thus, we will review this black hole solution in the present section. We begin with the action of five-dimensional Einstein-Maxwell-dilaton gravity (we use the convention $\kappa^{2}=8 \pi G$ ),

$$
\begin{aligned}
S_{\mathrm{EMD}}= & \frac{1}{2 \kappa_{5}^{2}} \int_{M} d^{5} x \sqrt{-g}\left[\mathcal{R}-\frac{4}{3} g^{M N} \partial_{M} \phi \partial_{N} \phi\right. \\
& \left.-V(\phi)-e^{-\frac{4}{3} \alpha \phi} F^{2}\right],
\end{aligned}
$$

where $\mathcal{R}$ and $\phi$ are the usual Ricci scalar and dilaton field, respectively; the latter has self-interaction $V(\phi)$ and nonminimally couples to the electromagnetic field of kinetic energies $F^{2}$. The physical constant $\alpha$ measures the strength of this coupling. Equation of motions following from this action have the form

$$
\begin{aligned}
\mathcal{R}_{M N}= & \frac{1}{3}\left[4 \partial_{M} \phi \partial_{N} \phi+g_{M N} V(\phi)\right] \\
& +2 e^{-\frac{4 \alpha \phi}{3}}\left[F_{M}^{L} F_{L N}-\frac{1}{6} g_{M N} F^{2}\right],
\end{aligned}
$$




$$
\begin{gathered}
\nabla^{2} \phi=\frac{\partial_{M}\left(\sqrt{-g} g^{M N} \partial_{N} \phi\right)}{\sqrt{-g}}=\frac{3}{8} \frac{\partial V}{\partial \phi}-\frac{\alpha}{2} e^{-\frac{4 \alpha \phi}{3}} F^{2}, \\
\nabla_{N}\left(e^{-\frac{4 \alpha \phi}{3}} F^{N M}\right)=\partial_{N}\left(\sqrt{-g} e^{-\frac{4 \alpha \phi}{3}} F^{N M}\right)=0 .
\end{gathered}
$$

We consider a static black hole solution with the metric ansatz,

$$
\begin{aligned}
d s^{2} & =g_{A B} d x^{A} d x^{B} \\
& =-A(r) d t^{2}+B(r) d r^{2}+R(r)^{2} d \Omega_{k, 3}^{2},
\end{aligned}
$$

where $d \Omega_{k, 3}^{2}$ is the line element of three-dimensional hypersurface of constant curvature $6 k$ with $k= \pm 1,0$ corresponding to spheric, hyperbolic, and plane topology, respectively. In the case of only static electric fields that occur in the system, the only nonzero components of $F_{M N}$ could be obtained from the Maxwell equation (4),

$$
F_{t r}=\sqrt{A(r) B(r)} \frac{q e^{4 \alpha \phi / 3}}{R^{3}(r)} .
$$

Explicitly, by substituting (5), (4) into (2) and (3), we obtain

$$
\begin{array}{r}
\frac{A^{\prime \prime}}{2 B}+\frac{3 A^{\prime} R^{\prime}}{2 B R}-\frac{A^{\prime} B^{\prime}}{4 B^{2}}-\frac{\left(A^{\prime}\right)^{2}}{4 A B}=\frac{4 e^{-4 \alpha \phi / 3}\left(F_{t r}\right)^{2}}{3 B}-\frac{1}{3} A V, \\
\begin{aligned}
\frac{A^{\prime \prime}}{2 A}+\frac{3 R^{\prime \prime}}{R}-\frac{3 B^{\prime} R^{\prime}}{2 B R}-\frac{A^{\prime} B^{\prime}}{4 A B}-\frac{\left(A^{\prime}\right)^{2}}{4 A^{2}}= & -\frac{1}{3} B V-\frac{4}{3}\left(\phi^{\prime}\right)^{2} \\
& +\frac{4\left(F_{t r}\right)^{2} e^{-4 \alpha \phi / 3}}{3 A},
\end{aligned}
\end{array}
$$

$$
\begin{aligned}
\frac{R R^{\prime \prime}}{B}+\frac{2\left(R^{\prime}\right)^{2}}{B}-\frac{B^{\prime} R^{\prime} R}{2 B^{2}}+\frac{A^{\prime} R^{\prime} R}{2 A B}= & -\frac{1}{3} R^{2} V \\
& -\frac{2 R^{2}\left(F_{t r}\right)^{2} e^{-4 \alpha \phi / 3}}{3 A B},
\end{aligned}
$$

$\frac{\phi^{\prime \prime}}{B}+\frac{3 R^{\prime} \phi^{\prime}}{\mathrm{BR}}-\frac{B^{\prime} \phi^{\prime}}{2 B^{2}}+\frac{A^{\prime} \phi^{\prime}}{2 A B}=\frac{3}{8} V^{\prime}+\frac{\alpha\left(F_{t r}\right)^{2} e^{-4 \alpha \phi / 3}}{A B}$,

where we have set $k=1$ with topology $S^{3}$, namely $d \Omega_{3}^{2}=d \theta_{1}^{2}+\sin ^{2} \theta_{1} d \theta_{2}^{2}+\sin ^{2} \theta_{1} \sin ^{2} \theta_{2} d \varphi^{2}$. For simplicity, we will consider only the spherical case in this paper. By adjusting the form of $V(\phi)$ appropriately, Refs. [48,49] obtain asymptotically AdS black hole solutions of the system analytically,

$$
\begin{gathered}
V(\phi)=\frac{\Lambda}{2\left(2+\alpha^{2}\right)^{2}}\left(4 \alpha^{2}\left(\alpha^{2}-1\right) \cdot e^{-\frac{8 \phi}{3 \alpha}}\right. \\
\left.+4\left(4-\alpha^{2}\right) \cdot e^{\frac{4 \alpha \phi}{3}}+24 \alpha^{2} \cdot e^{-\frac{2\left(2-\alpha^{2}\right) \phi}{3 \alpha}}\right), \\
A(r)=-\frac{c^{2}}{r^{2}}\left(1-\frac{b^{2}}{r^{2}}\right)^{1-\frac{2 \alpha^{2}}{2+\alpha^{2}}}-\frac{\Lambda r^{2}}{6}\left(1-\frac{b^{2}}{r^{2}}\right)^{\frac{\alpha^{2}}{2+\alpha^{2}}}, \\
B(r)=\left(1-\frac{b^{2}}{r^{2}}\right)^{-\frac{\alpha^{2}}{2+\alpha^{2}}} / A(r), \\
R(r)=\left(1-\frac{b^{2}}{r^{2}}\right)^{\frac{\alpha^{2}}{2\left(2+\alpha^{2}\right)}} r \\
\phi(r)=\frac{3 \alpha}{2\left(2+\alpha^{2}\right)} \ln \left(1-\frac{b^{2}}{r^{2}}\right) .
\end{gathered}
$$

Besides, the following relation is also implied by the Einstein field equations:

$$
q^{2}=\frac{6}{\left(2+\alpha^{2}\right)} b^{2} c^{2}
$$

Here $b$ and $c$ are integration constants with dimension of length, as we show later; they are also related with the mass $M$. Reference [50] shows that for this black hole solution, the Kretschmann scalar $R^{\mu \nu \alpha \beta} R_{\mu \nu \alpha \beta}$ and the Ricci scalar R both diverge at $r=b$; thus, $r=b$ is the location of curvature singularity. Furthermore, as shown in Fig. 1, for AdS black hole solution, there only exists one horizon whatever the value of dilaton coupling constant $\alpha$.

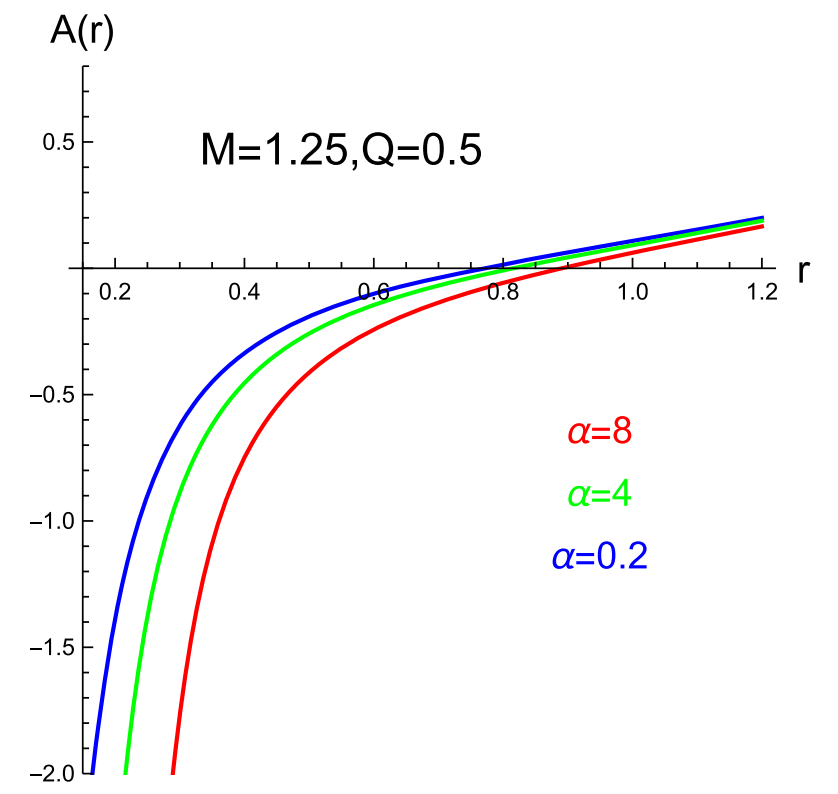

FIG. 1. Plot the horizon function $A(r)$ of a charged AdS dilaton black hole solution at a fixed black hole mass and charge with a different dilaton coupling constant. 


\section{B. The mass of black holes}

When we consider the black hole thermodynamics through Euclidean path integral approach, there exists an unavoidable divergence on the boundary of spacetime. In asymptotically AdS spacetime, a suitable surface counterterm can be found with the feature of coordinate frame independence; after the renormalization procedure, a finite Euclidean action and a well-defined boundary stress-energy tensor will be obtained. According to the methods of Refs. [59-61], we choose the surface counterterm as the following ansatz:

$$
\begin{aligned}
S_{c t}= & -\frac{1}{\kappa_{5}^{2}} \int_{\partial M} d^{4} x \sqrt{-\gamma}\left\{\frac{c_{0}}{l_{\mathrm{eff}}(\phi)}\left(1+\frac{c_{\phi}}{c_{0}} \phi^{2}\right)\right. \\
& \left.+c_{1} l_{\mathrm{eff}} \mathcal{R}+c_{2} l_{\mathrm{eff}}^{3}\left(\mathcal{R}^{2}+c_{\beta} \mathcal{R}^{\mathrm{ab}} \mathcal{R}_{\mathrm{ab}}\right)\right\},
\end{aligned}
$$

where $\frac{1}{l_{\text {eff }}(\phi)}=\sqrt{-\frac{V(\phi)}{3(3+1)}}$,

the expression of $V(\phi)$ is shown in Eq. (11). Actually, $\mathcal{R}^{2}$ and $\mathcal{R}^{a b} \mathcal{R}_{a b}$ are similar in magnitude, and we will make $c_{\beta}=0$ for simplification in calculation. With inclusion of this counterterm, the quasilocal stress-energy tensor at the boundary $r=$ const with induced metric $\gamma_{a b}$ could be derived as

$$
\begin{aligned}
T_{a b}= & \frac{1}{\kappa_{5}^{2}}\left\{K_{a b}-K \gamma_{a b}+\frac{c_{0}}{l_{\mathrm{eff}}}\left(1+c_{\phi} \phi^{2}\right) \gamma_{a b}\right. \\
& -2 c_{1} l_{\mathrm{eff}}\left(\mathcal{R}_{a b}-\frac{1}{2} \mathcal{R} \gamma_{a b}\right)+c_{2} l_{\mathrm{eff}}^{3}\left(\gamma_{a b} \mathcal{R}^{2}\right. \\
& \left.\left.-4 \mathcal{R}_{a b}+4 \nabla_{a} \nabla_{b} \mathcal{R}-4 \gamma_{a b} \nabla_{m} \nabla^{m} \mathcal{R}\right)\right\},
\end{aligned}
$$

where $\gamma_{a b}$ is the induced metric on the boundary $r=$ const, which is defined as

$\gamma_{a b} d x^{a} d x^{b}=\lim _{r \rightarrow \text { con }} d s_{5}^{2}=-A(r) d t^{2}+(R(r))^{2} d \Omega_{3}^{2}$.

The $K_{a b}$ is the extrinsic curvature on the boundary. By expanding (18) explicitly, we get

$$
\begin{gathered}
T_{t t}=-3 \frac{A R^{\prime}}{\sqrt{B} R}-\frac{c_{0}}{l_{\mathrm{eff}}} A\left(1+c_{\phi} \phi^{2}\right) \\
-6 \frac{c_{1} l_{\mathrm{eff}}}{R^{2}} A-36 \frac{c_{2} l_{\mathrm{eff}}^{3}}{R^{4}} A, \\
T_{i j}=\frac{h_{i j}}{R^{2}}\left(\frac{R^{2} A^{\prime}}{2 A \sqrt{B}}+2 \frac{R R^{\prime}}{\sqrt{B}}+c_{0}\left(1+\frac{c_{\alpha}}{c_{0}} \phi^{2}\right) \frac{R^{2}}{l_{\mathrm{eff}}}\right. \\
\left.+2 c_{1} l_{\mathrm{eff}}-12 c_{2} \frac{l_{\mathrm{eff}}^{3}}{R^{2}}\right) .
\end{gathered}
$$

The mass of a black hole is a conserved charge associated with a timelike killing vector; from Ref. [59], it could be defined as

$$
M=\int_{r \rightarrow \infty} d x^{3}(R(r))^{3}(A(r))^{-\frac{1}{2}} T_{t t} .
$$

For getting a finite mass of the black hole, we need to find a well-defined quasilocal stress-energy $T_{t t}$. Thus, we choose the undetermined coefficients in (17) as $c_{0}=-3, c_{\phi}=\frac{2}{9}$, $c_{1}=-\frac{1}{4}, c_{2}=\frac{1}{96}$. After substituting these coefficients and (11)-(15) into (22), we obtain

$$
M=\frac{3 \Omega_{3}}{2 \kappa_{5}^{2}}\left(c^{2}+\left(\frac{2-\alpha^{2}}{2+\alpha^{2}}\right) b^{2}\right) .
$$

\section{The thermodynamical quantities of black hole}

In terms of the general metric ansatz (5), the Hawking temperature could be expressed as

$$
T_{H}=\left.\frac{\sqrt{A^{\prime}\left(B^{-1}\right)^{\prime}}}{4 \pi}\right|_{r=r_{+}} .
$$

Substituting (12) and (13) into (24), we obtain

$$
\begin{aligned}
T_{H}= & \frac{\left(\left(2+\alpha^{2}\right) r^{2}-3 b^{2}\right)}{\left(\alpha^{2}+2\right) \pi L^{2} r_{+}}\left(1-\frac{b^{2}}{r_{+}^{2}}\right)^{\frac{\alpha^{2}-4}{2\left(2+\alpha^{2}\right)}} \\
& +\frac{1}{2 \pi r_{+}}\left(1-\frac{b^{2}}{r_{+}^{2}}\right)^{\frac{4-\alpha^{2}}{2\left(2+\alpha^{2}\right)}}
\end{aligned}
$$

in which we have used $A\left(r_{+}\right)=0$ to eliminate the parameter $c$, namely

$$
c=\left(1+\left(1-\frac{b^{2}}{r_{+}^{2}}\right)^{\frac{2 \alpha^{2}-2}{2+\alpha^{2}}} \frac{r_{+}^{2}}{L^{2}}\right)^{1 / 2} r_{+} .
$$

According to area laws, the entropy of system is

$$
S=\frac{2 \pi r_{+}^{3} \Omega_{3}}{\kappa_{5}^{2}}\left(1-\frac{b^{2}}{r_{+}^{2}}\right)^{\frac{32^{2}}{2\left(2+\alpha^{2}\right)}} .
$$

From the Gauss law, the electric charge is

$$
Q=\frac{b r_{+} \Omega_{3}}{4 \pi} \sqrt{\frac{6}{2+\alpha^{2}}} \sqrt{1+\frac{r_{+}^{2}}{L^{2}}\left(1-\frac{b^{2}}{r_{+}^{2}}\right)^{\frac{2 \alpha^{2}-2}{2+\alpha^{2}}}},
$$

in which we also use equality $A\left(r_{+}\right)=0$ to eliminate parameter $c$. Finally, the chemical potentials can be calculated as 


$$
U=\left.A_{t}\right|_{r \rightarrow \infty}-\left.A_{t}\right|_{r \rightarrow r_{+}}=-\frac{4 \pi q}{\kappa_{5}^{2} r_{+}^{2}},
$$

where the definition of $A_{t}$ is

$$
A_{t}=-\int d r \frac{8 \pi}{\kappa^{2}} F_{t r}=-\int d r \frac{8 \pi q}{\kappa^{2} r^{3}}=\frac{8 \pi}{2 \kappa^{2}} \frac{q}{r^{2}} .
$$

Replacing the parameter $c$ in (23) with the equality (26), we get

$$
M=\frac{3 \Omega_{3} r_{+}^{2}}{2 \kappa_{5}^{2}}\left(1+\left(1-\frac{b^{2}}{r_{+}^{2}}\right)^{\frac{2 \alpha^{2}-2}{2+\alpha^{2}}} \frac{r_{+}^{2}}{L^{2}}+\left(\frac{2-\alpha^{2}}{2+\alpha^{2}}\right) \frac{b^{2}}{r_{+}^{2}}\right) .
$$

With thermodynamic quantities given above, we can ensure the rightness of black hole mass (23) by checking that the first law of black hole thermodynamics is holden. According to the link rule of differentiation [50,53], we obtain

$$
\begin{gathered}
\left(\frac{\partial M}{\partial S}\right)_{Q}=\left(\frac{\partial M}{\partial r_{+}} \frac{\partial r_{+}}{\partial b}+\frac{\partial M}{\partial b}\right)_{Q} /\left(\frac{\partial S}{\partial r_{+}} \frac{\partial r_{+}}{\partial b}+\frac{\partial S}{\partial b}\right)_{Q} \\
\left(\frac{\partial r_{+}}{\partial b}\right)_{Q}=-\left(\frac{\partial Q}{\partial b}\right) /\left(\frac{\partial Q}{\partial r_{+}}\right), \\
\left(\frac{\partial M}{\partial Q}\right)_{S}=\left(\frac{\partial M}{\partial r_{+}} \frac{\partial r_{+}}{\partial b}+\frac{\partial M}{\partial b}\right)_{S} /\left(\frac{\partial Q}{\partial r_{+}} \frac{\partial r_{+}}{\partial b}+\frac{\partial Q}{\partial b}\right)_{S} \\
\left(\frac{\partial r_{+}}{\partial b}\right)_{S}=-\left(\frac{\partial S}{\partial b}\right) /\left(\frac{\partial S}{\partial r_{+}}\right) .
\end{gathered}
$$

Note that the extra minus sign in (33) and (35) originated from the derivative of implicit functions $Q\left(b, r_{+}\right)=$ $Q_{0}, S\left(b, r_{+}\right)=S_{0}$. After some tedious but straightforward calculations, it is easy to check that

$$
\left(\frac{\partial M}{\partial S}\right)_{Q}=T, \quad\left(\frac{\partial M}{\partial Q}\right)_{S}=U,
$$

thus the above thermodynamics quantities indeed satisfy the first law of black hole thermodynamics,

$$
d M=T d S+U d Q
$$

\section{BRANE'S MOTION IN SPACETIME OF BLACK HOLE}

\section{A. Motion of brane in a static bulk with one extra dimension}

We assume $\mathcal{M}$ is a five-dimensional manifold containing a brane $\Sigma$ with two sides (denoted by $\Sigma_{ \pm}$, respectively),
TABLE I. This table shows how to embed brane into bulk in each component of coordinates.

\begin{tabular}{lccc}
\hline \hline Bulk & $t$ & $r$ & $\vec{X}_{3}$ \\
Brane & $t_{b}$ & $\boldsymbol{x}$ & $\vec{x}_{3}$ \\
Embedded brane & $t\left(t_{b}\right)$ & $r\left(t_{b}\right)$ & $\vec{x}_{3}=\vec{X}_{3}$ \\
\hline \hline
\end{tabular}

which splits $\mathrm{M}$ into two parts, i.e., $\mathcal{M}_{ \pm}$, with a $Z_{2}$ symmetry. For describing the bulk-brane system clearly, we need to introduce some physical quantities before giving formula expression. We use $X^{A}$ to denote bulk coordinates and $x^{\mu}$ to denote internal coordinates of the brane world sheet. If we consider the motion of brane in bulk with one extra dimension, one can then embed brane into bulk with trajectory $X^{A}\left(x^{\mu}\right)$ (see Table I for more details) while constructing the vielbein as $e_{\mu}^{A}=\frac{\partial X^{A}}{\partial x^{\mu}}$. According to the static gauge, $t_{b}$ could be chosen as proper time $\tau$ of bulk coordinates. We will set $t_{b}=\tau$ in the following contents.

We consider a static bulk spacetime with the ansatz (5). The velocity of the brane could be written as $u^{M}=$ $(\dot{T}(\tau), \dot{r}(\tau), 0,0,0)$; normalization $u^{M} u_{M}=-1$ gives $\dot{T}(\tau)=$ $\sqrt{\left(1+B \dot{r}^{2}\right) / A}$. Let $n_{M}$ be the unit normal point into $M_{ \pm}$; according to orthogonal condition $u^{M} n_{M}=0, n^{M} n_{M}=1$, we obtain $n_{M}=\left(\sqrt{A B} \dot{r},-\sqrt{B\left(1+B \dot{r}^{2}\right)}, 0,0,0\right)$. The induced metric on $\Sigma_{ \pm}$is defined by $h_{\mu \nu}=e_{\mu}^{M} e_{\mu}^{N} g_{A B}$; explicitly,

$$
d s^{2}=-d \tau^{2}+R(r(\tau))^{2} d \Omega_{3}^{2}
$$

The physics of the bulk-brane system could be described by the action

$$
\begin{gathered}
S=S_{\text {EMD }}+S_{\text {brane }} \\
S_{\text {brane }}=\int d x^{4} \sqrt{-h}\left\{\frac{\mathcal{K}}{\kappa_{5}^{2}}+\lambda(\phi)+\mathcal{L}_{\text {matter }}\right\},
\end{gathered}
$$

where $\lambda$ is an undetermined function of $\phi$, which represents the effective brane tension. And $\mathcal{K}$ is the trace of the extrinsic curvature tensor [62]. Varying (39) with respect to metric tensor, besides getting a standard Einstein equation in bulk spacetime, we also obtain an Israel junction condition on the brane $[19,58]$,

$$
\left.\left\{\mathcal{K}_{\mu \nu}-\mathcal{K} h_{\mu \nu}\right\}\right|_{\Sigma_{+}}-\left.\left\{\mathcal{K}_{\mu \nu}-\mathcal{K} h_{\mu \nu}\right\}\right|_{\Sigma_{-}}=\kappa_{5}^{2} S_{\mu \nu},
$$

where $S_{\mu \nu}$ is the energy-momentum tensor of matters which are confined to the brane, and $\mathcal{K}_{\mu \nu}$ is defined as

$$
\mathcal{K}_{\mu \nu}=\frac{1}{2} e_{\mu}^{M} e_{\nu}^{N}\left(\nabla_{M} n_{N}+\nabla_{N} n_{M}\right)
$$


We will involve a $Z_{2}$ symmetry on two sides of the brane, namely $\left.\left\{\mathcal{K}_{\mu \nu}\right\}\right|_{\Sigma_{-}}=-\left.\left\{\mathcal{K}_{\mu \nu}\right\}\right|_{\Sigma_{+}}$. In this way, the junction condition (41) could be further simplified as

$$
\mathcal{K}_{\mu \nu}-\mathcal{K} h_{\mu \nu}=\frac{\kappa_{5}^{2}}{2} \mathcal{S}_{\mu \nu}
$$

for clearness in the expression; we omit the subscript $\Sigma_{+}$. Alternatively, (43) could also be written as

$$
\mathcal{K}_{\mu \nu}=-\frac{\kappa_{5}^{2}}{2}\left(S_{\mu \nu}-\frac{1}{3} S h_{\mu \nu}\right) .
$$

For general ansatz of a five-dimensional static black hole (5), the nonvanishing components of $\mathcal{K}_{\mu \nu}$ are given by

$$
\begin{gathered}
\mathcal{K}_{i j}=-\frac{R^{\prime}}{R} \frac{\sqrt{1+B \dot{r}^{2}}}{\sqrt{B}} h_{i j}, \\
\mathcal{K}_{\tau \tau}=\frac{\sqrt{\left(1+B \dot{r}^{2}\right)}}{2 A \sqrt{B}}\left(A^{\prime}+\left(A B^{\prime}-A^{\prime} B\right) \dot{r}^{2}\right),
\end{gathered}
$$

in which $\dot{r}=d r(\tau) / d \tau$, and we will also use the dot to denote the derivative with respect to $\tau$ in the following contents. Varying (39) with respect to the dilaton field. Besides giving the equation of motions (10) in bulk, we also obtain a boundary condition of dilaton field on the brane,

$$
\frac{4}{3 \kappa_{5}^{2}} n^{M} \partial_{M} \phi=\frac{\partial \lambda}{\partial \phi}
$$

Expanding (47) yields

$$
-\frac{\sqrt{\left(1+B \dot{r}^{2}\right)}}{\sqrt{B}} \partial_{r} \phi=\frac{3 \kappa_{5}^{2}}{4} \frac{\partial \lambda}{\partial \phi} .
$$

Besides, if we set the energy-momentum tensor of matters on the brane as simple ideal fluid types, namely

$$
\mathcal{S}_{\nu}^{\mu}=\operatorname{Diag}\{-\rho, P, P, P\},
$$

then by combining (49) with (40), we will obtain two independent components of the Israel junction condition as

$$
\begin{gathered}
\frac{\sqrt{\left(1+B \dot{r}^{2}\right)}}{2 A \sqrt{B}}\left(A^{\prime}+\left(A B^{\prime}-A^{\prime} B\right) \dot{r}^{2}\right)+\frac{2 R^{\prime} \sqrt{1+B \dot{r}^{2}}}{R \sqrt{B}} \\
=\frac{\kappa_{5}^{2}}{2}(\lambda+P), \\
\frac{R^{\prime}}{R} \frac{\sqrt{1+B \dot{r}^{2}}}{\sqrt{B}}=\frac{\kappa_{5}^{2}}{6}(\lambda-\rho) .
\end{gathered}
$$

\section{B. Effective Einstein field equations on the brane}

As shown in [13], the effective Einstein field equation on the brane is

$$
\begin{gathered}
\frac{3}{2}\left(\mathcal{R}_{\mu \nu}-\frac{1}{6} h_{\mu \nu} \mathcal{R}\right)+\frac{3}{2} E_{\mu \nu}-\frac{3}{2} \mathcal{K} \mathcal{K}_{\mu \nu}+\frac{3}{2} \mathcal{K}_{\mu \rho} \mathcal{K}_{\nu}^{\rho}+\frac{1}{4} h_{\mu \nu} \mathcal{K}^{2} \\
-\frac{1}{4} h_{\mu \nu} \mathcal{K}_{\alpha \beta} \mathcal{K}^{\alpha \beta}=\kappa_{5}^{2}\left(\mathcal{T}_{M N} h_{\mu}^{M} h_{\nu}^{N}-\frac{1}{4} h_{\mu \nu} \mathcal{T}\right)
\end{gathered}
$$

in which $R_{\mu \nu}$ and $R$ are the Ricci tensor and Ricci scalar of the induced metric $h_{\mu \nu}$, while $E_{\mu \nu}$ is defined as

$$
E_{\mu \nu}=\mathcal{C}_{M N A B} n^{M} n^{A} e_{\mu}^{N} e_{\nu}^{B}
$$

where $\mathcal{C}$ is the Weyl curvature tensor,

$$
\mathcal{C}_{M N A B}=R_{M N A B}-\frac{2}{3}\left(g_{M A} R_{B N}-g_{N A} R_{B M}\right)+\frac{1}{6} g_{M A} g_{B N} R .
$$

Substituting (38), (43) into (52) and expanding it explicitly, we obtain two equations as follows:

$$
\begin{gathered}
\frac{3 A^{\prime}}{2 A B} \frac{R^{\prime}}{R}+\frac{3 A^{\prime}}{2 A} \frac{R^{\prime}}{R} \dot{r}^{2}+\frac{3 B^{\prime}}{2 B} \frac{R^{\prime}}{R} \dot{r}^{2}-\frac{3}{2 B} \frac{\left(R^{\prime}\right)^{2}}{R^{2}}-\frac{3}{2} \frac{\left(R^{\prime}\right)^{2}}{R^{2}} \\
+3 \frac{R^{\prime}}{R} \ddot{r}=\frac{\kappa_{5}^{4} \lambda}{4} P+\frac{\kappa_{5}^{4} \lambda^{2}}{24}-\frac{\kappa_{5}^{4}}{4} P \rho+\frac{\kappa_{5}^{4} \lambda}{6} \rho-\frac{5 \kappa_{5}^{4}}{24} \rho^{2},
\end{gathered}
$$

$$
\frac{3}{2 B} \frac{\left(R^{\prime}\right)^{2}}{R^{2}}\left(1+B \dot{r}^{2}\right)=\frac{\kappa_{5}^{4} \lambda^{2}}{24}-\frac{\kappa_{5}^{4} \lambda}{12} \rho+\frac{\kappa_{5}^{4}}{24} \rho^{2} .
$$

Note that $R$ represents the solution (14), which is the function of $r$ in metric (5) rather than the Ricci scalar. It is easy to see that (55) is same with (51). Thus, the bulk-brane system is described by four independent equations (48), (50), (51), and (54). When combining with the Einstein field equation in bulk (7)-(9) and the Israel junction condition (50) and (51), (54) could be further simplified as

$$
\ddot{r}+\frac{A^{\prime}}{2 A} \dot{r}^{2}+\frac{A^{\prime} B}{2 A} \dot{r}^{4}-\frac{1}{2} B^{\prime} \dot{r}^{4}=0,
$$

after substituting (12), (13) into (56) and solving this differential equation numerically at fixed parameters $M$ and $Q$ with different $\alpha$. We display the variation of brane's position $r(\tau)$ and the corresponding velocity $\dot{r}(\tau)$ in the topleft and the top-right panels of Fig. 2. Note that for the observer confined on the brane universe, the scale factor is $R(r(\tau))$ according to the induced metric (38); thus, we also show the variation trend of $R(r(\tau))$ and the corresponding Hubble constant $H=\frac{\dot{R}}{R}=\frac{R^{\prime}(r(\tau))}{R} \dot{r}$ in Fig. 2.

From (48), we obtain the mathematical expression of the brane tension $\lambda$ as the integrate function of $\tau$, 

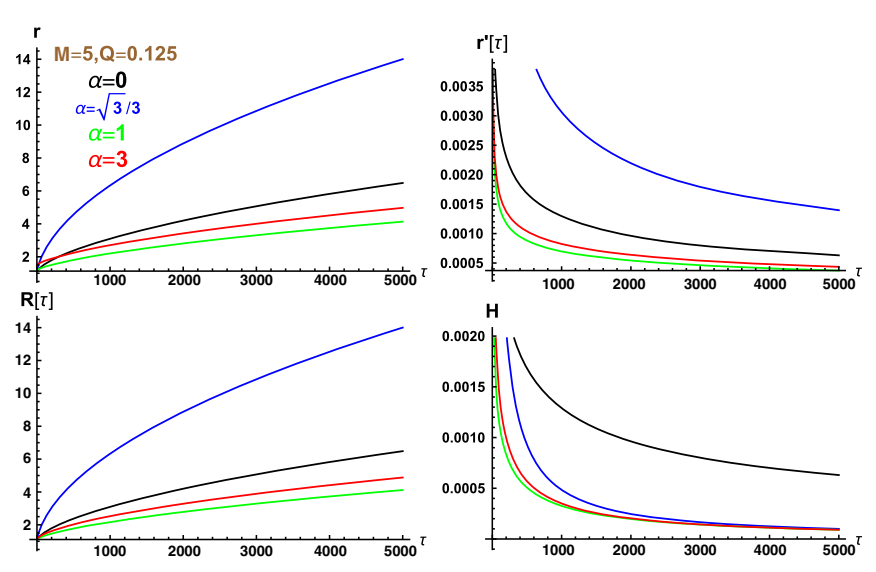

FIG. 2. The upper part displays the location $r(\tau)$ and the velocity $\frac{d r(\tau)}{d \tau}$ of the brane in bulk coordinates as the increase of proper time. The bottom part plots the evolution of effective scale factor $R(r(\tau))$ and the Hubble constant $H=\frac{d R / d \tau}{R}$ for the observer confined on the brane universe. The red, green, blue, and black curves correspond to $\alpha=3, \alpha=1, \alpha=\sqrt{3} / 3, \alpha=0$, respectively. Meanwhile, all of these curves correspond to the same black hole mass and charge. Both the AdS radius and $\kappa$ are set to be 1 .

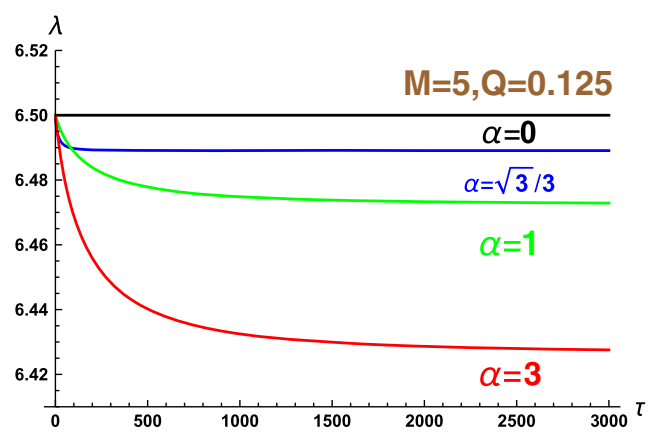

FIG. 3. According to Eq. (57), we plot the evolution of effective brane tension $\lambda$ as the function of proper time $\tau$. The initial brane's tension is set as $\lambda_{0}=6.5$. Both the AdS radius and $\kappa$ are set to be 1 .

$$
\lambda=\lambda_{0}-\frac{4}{3 \kappa_{5}^{2}} \int \frac{\sqrt{\left(1+B \dot{r}^{2}\right)}}{\sqrt{B}}\left(\phi^{\prime}\right)^{2} \dot{r} d \tau .
$$

Based on (57), we give the variation trend of $\lambda$ with respect to $\tau$ in Fig. 3. Combining Eqs. (50) and (51) with the numerical solutions $r(\tau)$ and $\lambda(\tau)$, we can also plot the evolution of energy density $\rho$ and pressure $P$ of the matters confined on the brane as follows.

\section{HOLOGRAPHIC EFFECTS ON BRANE}

\section{A. A renormalization procedure inspired by AdS/CFT}

As indicated in [4], the energy, entropy, and temperature of the CFT on boundary spacetime at high temperatures could be identified with the mass, Bekenstein-Hawking entropy, and Hawking temperature of the AdS black hole. Thus, the matters on the brane will have the CFT's feature when the brane approaches the AdS boundary. In bulk spacetime, the total energy is just the black hole mass (23), namely $E=M$. In case of large $r(\tau)$, the matter's energy on the brane is given by

$$
E=M \dot{t} \approx \frac{3 \Omega_{3}}{2 \kappa_{5}^{2}}\left(c^{2}+\frac{2-\alpha^{2}}{2+\alpha^{2}} b^{2}\right) \frac{L}{r} .
$$

Note that $E=M$ is obtained by using the bulk's time coordinate $t$. Nevertheless, an observer on the brane measures the total energy by using the time coordinate $\tau$; thus, we need to scale $E$ by $\dot{t}$. And the energy density is given by

$$
\rho=\frac{E}{V}=\frac{3}{2 \kappa_{5}^{2}}\left(c^{2}+\frac{2-\alpha^{2}}{2+\alpha^{2}} b^{2}\right) \frac{L}{r^{4}},
$$

where $V=(R(r(\tau)))^{3} \Omega_{3}$ is just the spatial volume of the brane; from (15), we know that $R(r(\tau))=r(\tau)$ in the large $r(\tau)$ limit. Meanwhile, we use $r$ to denote $r(\tau)$ in (58) and (59) for convenience, and this convention will still be used in this and the next section. Namely, without pointing out explicitly, $r$ will always represent $r(\tau)$ in Sec. IV. According to the first law of thermodynamics,

$$
T d S+U d Q=d E+P d V
$$

the pressure is obtained as

$$
P=-\frac{\partial E}{\partial V}=\frac{L}{2 \kappa_{5}^{2} r^{4}}\left(c^{2}+\frac{2-\alpha^{2}}{2+\alpha^{2}} b^{2}\right) .
$$

It is easy to check that $P=\frac{1}{3} \rho$. However, from Fig. 4 , we observe that $P=-\rho$ in region of large $r$.
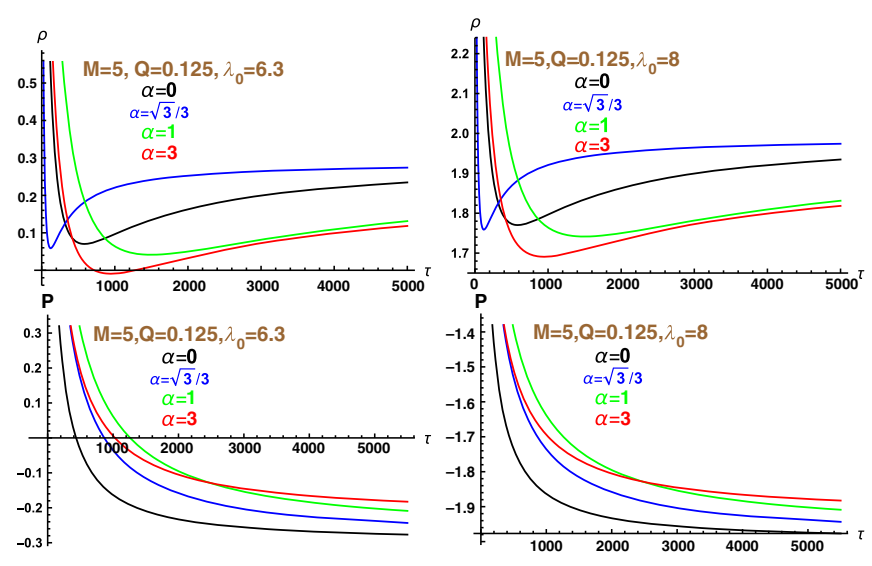

FIG. 4. The evolution of energy density $\rho$ and pressure $P$ for the matters confined on the brane, at fixed black hole mass $M$ and charge $Q$ with different dilaton coupling constant $\alpha$ and the initial brane tension $\lambda_{0}$. Both the AdS radius and $\kappa$ are set to be 1 . 
Actually, from the scenario of brane-bulk geometry, there exists a motional brane which has cut off the bulk spacetime. Translate the scenario of geometry into the word of quantum field theory, it means that the matter field confined on the brane is a symmetry broken effective field theory of CFT on the AdS boundary at lower energies. Namely, in the case of $r(\tau) \ll \infty$, the Israel junction could be reexpressed as

$$
\mathcal{K}_{\mu \nu}-\mathcal{K} h_{\mu \nu}=\frac{\kappa_{5}^{2}}{2}\left(\left\langle S_{\mu \nu}^{\mathrm{CFT}}\right\rangle+\left\langle S_{\mu \nu}^{\mathrm{IR}}\right\rangle+\lambda h_{\mu \nu}\right) .
$$

When the brane approaches the AdS boundary, namely $r(\tau) \rightarrow \infty$, we need to add the counterterms to subtract the infrared (IR) divergences and restore the conformal symmetry; finally, the modified Israel junction condition becomes

$$
\mathcal{K}_{\mu \nu}-\mathcal{K} h_{\mu \nu}-\frac{\kappa_{5}^{2}}{2} S_{\mu \nu}^{c t}=\frac{\kappa_{5}^{2}}{2}\left(\left\langle S_{\mu \nu}^{\mathrm{CFT}}\right\rangle+\lambda h_{\mu \nu}\right) .
$$

As considered in [63-65], the action of counterterms could be involved as the Ricci scalar $R$ and higher order curvature terms with respect to the brane's induced metric $h_{\mu \nu}$. To be consistent with (17), we choose the counterterm as the following ansatz:

$$
S_{c t}=-\frac{1}{\kappa_{5}^{2}} \int_{\Sigma} d^{4} x \sqrt{-h}\left\{b_{1} l_{\mathrm{eff}} \mathcal{R}+b_{2} l_{\mathrm{eff}}^{3} \mathcal{R}^{2}\right\},
$$

where $l_{\text {eff }}(\phi)=\sqrt{-\frac{3(3+1)}{V(\phi)}}$. Similarly, we have ignored the $\mathcal{R}^{\mu \nu} \mathcal{R}_{\mu \nu}$ term for simplification in calculation, without loss of generality. From (64), the corresponding $S_{\mu \nu}^{c t}$ could be calculated as

$$
\begin{aligned}
S_{\mu \nu}^{c t}= & -\frac{2 c_{1} l_{\text {eff }}}{\kappa_{5}^{2}}\left(\mathcal{R}_{\mu \nu}-\frac{1}{2} \mathcal{R} h_{\mu \nu}\right)+\frac{c_{2}}{\kappa_{5}^{2}} l_{\text {eff }}^{3}\left\{h_{\mu \nu} \mathcal{R}^{2}\right. \\
& \left.-4 \mathcal{R} \mathcal{R}_{\mu \nu}+4 \nabla_{\nu} \nabla_{\mu} \mathcal{R}-4 h_{\nu \mu} \nabla_{\alpha} \nabla^{\alpha} \mathcal{R}\right\} .
\end{aligned}
$$

Meanwhile, we assume $\lambda(\phi)$ has the following mathematical form in case of large $r(\tau)$ :

$$
\lambda(\phi)=\frac{1}{\kappa_{5}^{2}} \frac{b_{0}}{l_{\mathrm{eff}}(\phi)}\left(1+\frac{b_{\alpha}}{b_{0}} \phi^{2}\right)
$$

the coefficients $b_{0}, b_{\alpha}, b_{1}, b_{2}$ will be decided later.

Substituting (66) and (65) into the modified Israel junction condition (63), and expanding $\rho, P$ in the case of large $r(\tau)$, we obtain

$$
\begin{aligned}
\kappa_{5}^{2} \rho= & \frac{\left(b_{0}-6\right)}{L}-\frac{3 L\left(1-2 b_{1}\right)}{r^{2}} \\
+ & \frac{3}{4 L\left(2+\alpha^{2}\right)^{2} r^{4}}\left\{\alpha^{2} b^{4}\left(3 b_{\alpha}-8\right)\right. \\
+ & \left(2+\alpha^{2}\right)^{2} L^{2}\left(4 c^{2}+\left(1+48 b_{2}\right) L^{2}\right) \\
+ & \left.8\left(2+\alpha^{2}\right)\left(1+\left(b_{1}-1\right) \alpha^{2}\right) b^{2} L^{2}\right\}+O\left(\frac{1}{r^{6}}\right), \\
\kappa_{5}^{2} P= & \frac{\left(b_{0}-6\right)}{L}+\frac{\left(1-2 b_{1}\right) L}{r^{2}} \\
& +\frac{1}{4 L^{2}\left(2+\alpha^{2}\right)^{2} r^{4}}\left\{3 \alpha^{2} b^{4} L\left(8-3 b_{\alpha}\right)\right. \\
& +\left(2+\alpha^{2}\right)^{2} L^{3}\left(4 c^{2}+\left(1+48 b_{2}\right) L^{2}\right) \\
& \left.\quad-8\left(2+\alpha^{2}\right)\left(b_{1} \alpha^{2}-1\right) b^{2} L^{3}\right\}+O\left(\frac{1}{r^{6}}\right) .
\end{aligned}
$$

Note that Eq. (56) is invariant, although the Israel junction condition is changed from (41) to (63). So, the evolution of $r(\tau)$ is still controlled by Eq. (56). It is easy to observe from Fig. 2 that $\dot{r}$ and higher order derivatives will approach 0 when $r(\tau) \rightarrow \infty$; thus, we have ignored all $O\left(\frac{1}{r^{2}}\right), O\left(\frac{1}{r^{4}}\right)$ terms which include the derivatives of $r(\tau)$. Finally, by comparing (67) and (68) with the expected results (59) and (61), we can give these undetermined coefficients as $b_{0}=6, b_{1}=\frac{1}{2}, b_{\alpha}=\frac{8}{3}, b_{2}=-\frac{1}{48}$.

Substituting $b_{0}=6, b_{\alpha}=\frac{8}{3}$ into the (66), we obtain an analytical expression for the effective brane tension as a function of dilaton field,

$$
\lambda(\phi)=\frac{1}{\kappa_{5}^{2}} \frac{6}{l_{\mathrm{eff}}(\phi)}\left(1+\frac{4}{9} \phi^{2}\right) .
$$

Next, we check that (69) is indeed valid in large $r(\tau)$ case. By combining (69) with Eq. (10) and the numerical solution of $r(\tau)$, we can plot the evolution of $\lambda$ with respect to the $\tau$ as shown by the dotted curves in Fig. 5 . Meanwhile, (57) is also displayed in Fig. 5 by solid curves. It is obviously shown that by adjusting the parameter $\lambda_{0}$ appropriately, (57) is consistent with (69) at late time. Besides the validity of (10) in large $r(\tau)$ case, Fig. 5 also reveals a physical fact that a fixed $\lambda_{0}$ is implied by the AdS/CFT correspondence.

\section{B. Cardy-Verlinde formula}

The entropy of $(1+1)$-dimensional CFT system could be given by a very famous formula, namely the Cardy formula [23]. By using the combination of AdS/CFT correspondence and thermodynamics of AdS-Schwarzschild black holes, $[22,66]$ have generalized the Cardy formula to the CFT system which lives in arbitrary-dimensional 


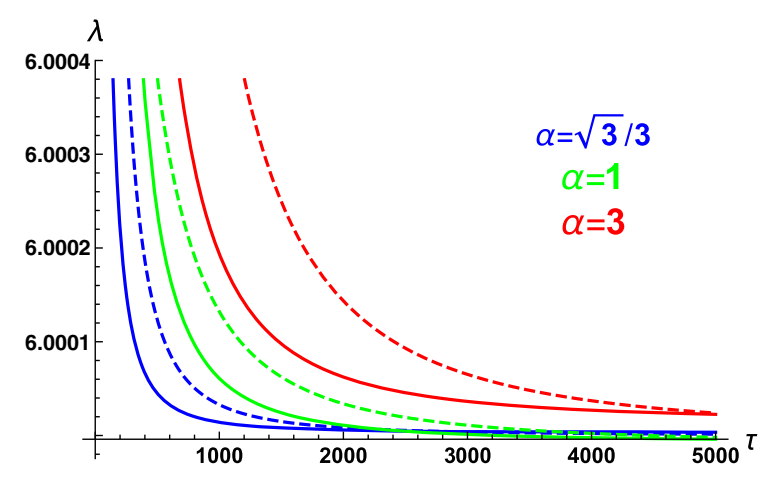

FIG. 5. The dotted curves correspond to the approximate solution of effective brane tension $\lambda$ given by (66) which is valid in large $r(\tau)$ case (as shown in Fig. $2, r(\tau)$ is the monotonically increasing function of $\tau$, and hence the large $r(\tau)$ value also means the large $\tau$ value). The solid curves correspond to the numerically exact solution of $\lambda$ given in (57). All these curves have the same parameter value $M=5, Q=0.125, L=1, \kappa=1$. The value of $\lambda_{0}$ set for the solid red, solid green, and solid blue curves are 6.0737, 6.02757, and 6.01093, respectively.

spacetime; this generalized Cardy formula is called the Cardy-Verlinde formula. After that, the Cardy-Verlinde formula has been checked in various black holes with AdS asymptotics [24-32,39]. In our case, as we have argued in the last section, the matters confined to the brane will behave as CFT system when the brane approaches the AdS boundary. Thus, we naturally expect that the CardyVerlinde formula will hold in case of large $r(\tau)$.

In the limit of $r(\tau) \rightarrow \infty$, the temperature and the chemical potential of the corresponding CFT on the brane could be given as

$$
\begin{aligned}
& T= T_{H} \dot{t} \approx \frac{L}{2 \pi r r_{+}}\left(1-\frac{b^{2}}{r_{+}^{2}}\right)^{\frac{4-\alpha^{2}}{2\left(2+\alpha^{2}\right)}} \\
&+ \frac{\left(\left(2+\alpha^{2}\right) r_{+}^{2}-3 b^{2}\right)}{\left(\alpha^{2}+2\right) \pi L r r_{+}}\left(1-\frac{b^{2}}{r_{+}^{2}}\right)^{\frac{\alpha^{2}-4}{2\left(2+\alpha^{2}\right)}}, \\
& U=U \dot{t} \approx-\frac{4 \sqrt{6} \pi b L}{\sqrt{2+\alpha^{2}} \kappa_{5}^{2} r r_{+}} \sqrt{1+\frac{r_{+}^{2}}{L^{2}}\left(1-\frac{b^{2}}{r_{+}^{2}}\right)^{\frac{2 \alpha^{2}-2}{2+\alpha^{2}}}} .
\end{aligned}
$$

The entropy and electric charge are given directly by (27) and (28). And we obtain the entropic and electric density as

$$
\begin{gathered}
s=\frac{S}{V}=\frac{2 \pi r_{+}^{3}}{\kappa_{5}^{2} r^{3}}\left(1-\frac{b^{2}}{r_{+}^{2}}\right)^{\frac{3 a^{2}}{2\left(2+\alpha^{2}\right)}}, \\
\rho_{e}=\frac{Q}{V}=\frac{\sqrt{6} b r_{+}}{4 \pi \sqrt{2+\alpha^{2}} r^{3}} \sqrt{1+\frac{r_{+}^{2}}{L^{2}}\left(1-\frac{b^{2}}{r_{+}^{2}}\right)^{\frac{2 \alpha^{2}-2}{2+\alpha^{2}}}} .
\end{gathered}
$$

Using the symbol in [22], the $\gamma$ quantity, which is relevant with the Casimir energy $E_{c}$ of CFT system on the brane through the relation $E_{c}=\left(\frac{\gamma}{r^{2}}\right) \cdot r^{3} \Omega_{3}$, could be defined as

$$
\begin{aligned}
\frac{\gamma}{r^{2}} & =3\left(\rho_{\mathrm{CFT}}+P_{\mathrm{CFT}}-\left|\rho_{e} U\right|-T s\right)-\frac{\gamma_{\text {proper }}}{r^{2}} \\
& =3 \frac{r_{+}^{2}}{\kappa_{5}^{2} r^{4}} L\left(1-\frac{b^{2}}{r_{+}^{2}}\right)
\end{aligned}
$$

where

$$
\frac{\gamma_{\text {proper }}}{r^{2}}=\frac{r_{+}^{2}}{\kappa_{5}^{2} r^{4}}\left(\frac{6}{2+\alpha^{2}} \frac{b^{2}}{r_{+}^{2}} L\right),
$$

the $\left(\frac{\gamma_{\text {proper }}}{r^{2}}\right) \cdot r^{3} \Omega_{3}$ has the physical meaning of proper internal energy which is viewed as the zero temperature energy of CFT [25]. Note also that the proper internal energy has no physical effects on the entropy; thus, it needs to be subtracted from the total energy when considering the Cardy-Verlinde formula. By combining the physical quantity $\gamma, \gamma_{\text {proper }}$ with the energy density $\rho$ in (59), it is easy to find that the entropy density of the brane universe in case of $r(\tau) \rightarrow \infty$ could be expressed as

$$
\left(\frac{3}{2 \pi} s\right)^{2}=\gamma\left(2\left(\rho_{\mathrm{CFT}}-\frac{\gamma_{\text {proper }}}{r^{2}}\right)-\frac{\gamma}{r^{2}}\right) .
$$

By comparing with the original version of the CardyVerlinde formula [22], our result (76) has an extra physical quantity $\gamma_{\text {proper }} / r^{2}$. As explained in [25], the $\gamma_{\text {proper }} / r^{2}$ is relevant with the proper internal energy which makes the contribution to the free energy of the CFT system, but not to the entropy. Thus, the contribution of the proper internal energy must be subtracted when we consider the relationship between the entropy and the energy of the CFT system on the brane. Besides, when $\alpha=0$, (76) will return the Cardy-Verlinde formula (3.16) in [25], which corresponds to the CFT system living on the boundary of the dS-RN black hole spacetime.

\section{CONCLUSION AND DISCUSSION}

In the background of a charged AdS dilaton black hole solved by [47-49], we study the movement of a selfgraviting brane and holographic effects as the brane gets close to the AdS boundary. Although the movement of the brane/wall in this AdS dilaton black hole has been investigated by the authors [54], they ignore the selfgravitating effects of the brane/wall. Specifically, they assume that there only exists matter field on the brane, and the evolution of brane is controlled by the Israel junction condition only. But a more realistic case is that the effective gravitational field should also be localized on the brane; thus, the evolution of brane is determined by the effective Einstein field equation and the Israel junction 
condition together. In our scenario, we derive the effective Einstein field equation on the brane by using the method provided by [13], in which they localize the gravitational field by projecting the bulk's Riemann curvature and its contractions (namely, the Ricci tensor and scalar curvature) on the brane spacetime. In results of [54], they obtained different evolution modes of the brane/wall in various physical parameters, especially for $\omega=\frac{P}{\rho}$ and dilaton coupling constant $\alpha$. However, when taking the inclusion of self-gravitating effects of the brane, we find that the evolution mode of the brane is not so sensitive to the value of parameters $\alpha$ and $\omega$. Specifically, as shown in Fig. 2, we observe the evolution of the brane only in the mode of decelerated expansion whatever the value of $\alpha$, while the velocity $\dot{r}$ of brane will tend to be zero as the brane gets close to the AdS boundary. On the other hand, from Fig. 4, it is obviously shown that $\omega=\frac{P}{\rho}$ will approach a fixed value -1 as time increases.

Inspired by AdS/CFT correspondence, we expect to observe a connection between the brane universe and the physical quantities of the AdS dilaton black hole when the brane gets close to the AdS boundary. Nevertheless, in our scenario of brane-bulk geometry, the AdS spacetime is cut off by a motional brane. And, the matter confined to the brane is described by a general quantum field theory without conformal symmetry. Thus, as the brane moves close to the boundary of the AdS dilaton black hole, a renormalization procedure should be considered to cancel the IR divergences and restore the conformal symmetry. Note that the IR divergences on the gravitational side correspond to the UV divergence on the CFT side. Furthermore, as indicated in [22], when the brane approaches the AdS boundary, AdS/CFT correspondence implies that a radiation dominated FLRW universe $\left(P=\frac{1}{3} \rho\right)$ should be given. We add an appropriate surface counterterm to the gravitational action by using the method given in [63-65]; after the inclusion of this counterterm, the correct energy density and pressure (namely $P=\frac{1}{3} \rho$ ) could be reproduced on the brane in the limit of $r(\tau) \rightarrow \infty$. It is remarkable that this surface counterterm also plays an important role in calculating the black hole mass. What is more, based on this holographic renormalization procedure, the evolution of brane's tension as the function of dilaton field could be given analytically in case of large $r(\tau)$.
By using a holographic method provided in [66], the temperature, entropy, charge, and chemical potential on the brane could be given from the corresponding thermodynamic quantities of the AdS dilaton black hole. With these thermodynamic quantities on the brane, an extended CardyVerlinde formula is obtained in the large $r(\tau)$ limit. Meanwhile, our result has a similar mathematical form with the one obtained in the background of the AdS-RN black hole [25]. This similarity is reasonable in physics, when making the dilaton coupling constant $\alpha=0$; the charged AdS dilaton black hole solution will return the AdS-RN one. Thus, when the brane approaches the AdS boundary, we naturally obtain a similar Cardy-Verlinde formula on the brane like the one in [25] but with some corrections of the dilaton field.

As a discussion, in light of this work, we point out that the following extensions are still worthwhile to explore. The first one is to investigate the holographic complexity growth in the FLRW brane and the black hole system constructed in this paper. Besides, as indicated in [40], in the frame of AdS/CFT correspondence, the reheating process in the brane universe could be dual to the collapse of a spherical shell and the formation of a black hole in AdS bulk. Thus, a valuable work is to study the holographic reheating in the current brane-bulk system. What is more, it is an interesting topic to consider the movement of brane in the background of asymptotically (A)dS black hole with scalar and electromagnetic hair [67-69] and check how the Cardy-Verlinde formula is affected by the scalar charge. Finally, it is also worthwhile to explore the movement of brane in the inner spacetime of some nonsingularity black hole solutions [70-73].

\section{ACKNOWLEDGMENTS}

A.-c.L. is grateful to Lei-Hua Liu and Carlos Herdeiro for fruitful discussions and comments on this research topic and paper, and thanks $\mathrm{Ru}$-Yong $\mathrm{Li}$ for her help in adjusting the physical parameters in a picture. This work is supported by NSFC Grant No. 11875082 . This work is also supported by the Center for Research and Development in Mathematics and Applications through the Portuguese Foundation for Science and Technology (FCT-Fundao para a Cincia e a Tecnologia), references UIDB/04106/ 2020 and UIDP/04106/2020.
[1] P. Horava and E. Witten, Heterotic and type I string dynamics from eleven-dimensions, Nucl. Phys. B460, 506 (1996).

[2] P. Horava and E. Witten, Eleven-dimensional supergravity on a manifold with boundary, Nucl. Phys. B475, 94 (1996).
[3] J. M. Maldacena, The large N limit of superconformal field theories and supergravity, Int. J. Theor. Phys. 38, 1113 (1999); Adv. Theor. Math. Phys. 2, 231 (1998).

[4] E. Witten, Anti-de Sitter space and holography, Adv. Theor. Math. Phys. 2, 253 (1998). 
[5] L. Randall and R. Sundrum, Out of this world supersymmetry breaking, Nucl. Phys. B557, 79 (1999).

[6] L. Randall and R. Sundrum, A Large Mass Hierarchy from a Small Extra Dimension, Phys. Rev. Lett. 83, 3370 (1999).

[7] L. Randall and R. Sundrum, An Alternative to Compactification, Phys. Rev. Lett. 83, 4690 (1999).

[8] V. A. Rubakov and M. E. Shaposhnikov, Do we live inside a domain wall?, Phys. Lett. 125B, 136 (1983).

[9] V. A. Rubakov and M. E. Shaposhnikov, Extra space-time dimensions: Towards a solution to the cosmological constant problem, Phys. Lett. 125B, 139 (1983).

[10] N. Arkani-Hamed, S. Dimopoulos, and G. R. Dvali, The hierarchy problem and new dimensions at a millimeter, Phys. Lett. B 429, 263 (1998).

[11] I. Antoniadis, N. Arkani-Hamed, S. Dimopoulos, and G. R. Dvali, New dimensions at a millimeter to a Fermi and superstrings at a TeV, Phys. Lett. B 436, 257 (1998).

[12] N. Arkani-Hamed, S. Dimopoulos, and G. R. Dvali, Phenomenology, astrophysics and cosmology of theories with submillimeter dimensions and $\mathrm{TeV}$ scale quantum gravity, Phys. Rev. D 59, 086004 (1999).

[13] T. Shiromizu, K. i. Maeda, and M. Sasaki, The Einstein equation on the 3-brane world, Phys. Rev. D 62, 024012 (2000).

[14] P. Bostock, R. Gregory, I. Navarro, and J. Santiago, Einstein Gravity on the Codimension 2-Brane?, Phys. Rev. Lett. 92, 221601 (2004).

[15] N. Kaloper, Bent domain walls as brane worlds, Phys. Rev. D 60, 123506 (1999).

[16] P. Kanti, I. I. Kogan, K. A. Olive, and M. Pospelov, Cosmological three-brane solutions, Phys. Lett. B 468, 31 (1999).

[17] E. E. Flanagan, S. H. H. Tye, and I. Wasserman, Cosmological expansion in the Randall-Sundrum brane world scenario, Phys. Rev. D 62, 044039 (2000).

[18] P. Kanti, T. Nakas, and N. Pappas, Antigravitating braneworld solutions for a de Sitter brane in scalar-tensor gravity, Phys. Rev. D 98, 064025 (2018).

[19] H. A. Chamblin and H. S. Reall, Dynamic dilatonic domain walls, Nucl. Phys. B562, 133 (1999).

[20] P. Kraus, Dynamics of anti-de Sitter domain walls, J. High Energy Phys. 12 (1999) 011.

[21] S. Creek, R. Gregory, P. Kanti, and B. Mistry, Braneworld stars and black holes, Classical Quantum Gravity 23, 6633 (2006).

[22] I. Savonije and E. P. Verlinde, CFT and entropy on the brane, Phys. Lett. B 507, 305 (2001).

[23] J. L. Cardy, Operator content of two-dimensional conformally invariant theories, Nucl. Phys. B270, 186 (1986).

[24] D. Klemm, A. C. Petkou, and G. Siopsis, Entropy bounds, monotonicity properties and scaling in CFTs, Nucl. Phys. B601, 380 (2001).

[25] R. G. Cai, The Cardy-Verlinde formula and AdS black holes, Phys. Rev. D 63, 124018 (2001).

[26] D. Birmingham and S. Mokhtari, The Cardy-Verlinde formula and Taub-Bolt-AdS space-times, Phys. Lett. B 508, 365 (2001).

[27] D. Youm, The Cardy-Verlinde formula and topological AdS-Schwarzschild black holes, Phys. Lett. B 515, 170 (2001).
[28] R. G. Cai and Y.Z. Zhang, Holography and brane cosmology in domain wall backgrounds, Phys. Rev. D 64, 104015 (2001).

[29] D. Youm, The Cardy-Verlinde formula and charged topological AdS black holes, Mod. Phys. Lett. A 16, 1327 (2001).

[30] J. 1. Jing, Cardy-Verlinde formula and entropy bounds in Kerr-Newman AdS(4)/dS(4) black hole backgrounds, Phys. Rev. D 66, 024002 (2002).

[31] R. G. Cai and Y. S. Myung, Holography and entropy bounds in Gauss-Bonnet gravity, Phys. Lett. B 559, 60 (2003).

[32] C. O. Lee, Cardy-Verlinde formula in Taub-NUT/Bolt-(A) dS space, Phys. Lett. B 670, 146 (2008).

[33] S. Nojiri and S. D. Odintsov, Can we live on the brane in Schwarzschild-anti-de Sitter black hole?, Phys. Lett. B 493, 153 (2000).

[34] S. Nojiri, S. D. Odintsov, and S. Ogushi, Holographic entropy and brane FRW dynamics from AdS black hole in d5 higher derivative gravity, Int. J. Mod. Phys. A 16, 5085 (2001).

[35] S. Nojiri, S. D. Odintsov, and S. Ogushi, FriedmannRobertson-Walker brane cosmological equations from the five-dimensional bulk (A)dS black hole, Int. J. Mod. Phys. A 17, 4809 (2002).

[36] J. E. Lidsey, S. Nojiri, S. D. Odintsov, and S. Ogushi, The AdS/CFT correspondence and logarithmic corrections to brane world cosmology and the Cardy-Verlinde formula, Phys. Lett. B 544, 337 (2002).

[37] S. Nojiri and S.D. Odintsov, Universal features of the holographic duality: Conformal anomaly and brane gravity trapping from 5-D AdS black hole, Int. J. Mod. Phys. A 18, 2001 (2003).

[38] S. Nojiri, S. D. Odintsov, and S. Ogushi, Logarithmic corrections to the FRW brane cosmology from 5-D Schwarzschild-de Sitter black hole, Int. J. Mod. Phys. A 18, 3395 (2003).

[39] M. Bravo Gaete, L. Guajardo, and M. Hassaine, A Cardylike formula for rotating black holes with planar horizon, J. High Energy Phys. 04 (2017) 092.

[40] S. Kawai and Y. Nakayama, Reheating of the Universe as holographic thermalization, Phys. Lett. B 759, 546 (2016).

[41] Y.S. An, R. G. Cai, L. Li, and Y. Peng, Holographic complexity growth in a FLRW universe, Phys. Rev. D 101, 046006 (2020).

[42] G. W. Gibbons and K.i. Maeda, Black holes and membranes in higher dimensional theories with Dilaton fields, Nucl. Phys. B298, 741 (1988).

[43] R. Gregory and J. A. Harvey, Black holes with a massive dilaton, Phys. Rev. D 47, 2411 (1993).

[44] J. H. Horne and G. T. Horowitz, Black holes coupled to a massive dilaton, Nucl. Phys. B399, 169 (1993).

[45] S. J. Poletti and D. L. Wiltshire, The global properties of static spherically symmetric charged dilaton space-times with a Liouville potential, Phys. Rev. D 50, 7260 (1994); S. J. Poletti and D. L. WiltshireErratum, Phys. Rev. D 52, 3753 (1995).

[46] S. Mignemi and D. L. Wiltshire, Black holes in higher derivative gravity theories, Phys. Rev. D 46, 1475 (1992).

[47] C. J. Gao and S. N. Zhang, Dilaton black holes in de Sitter or Anti-de Sitter universe, Phys. Rev. D 70, 124019 (2004). 
[48] C. J. Gao and S. N. Zhang, Higher dimensional dilaton black holes with cosmological constant, Phys. Lett. B 605, 185 (2005).

[49] C. J. Gao and S. N. Zhang, Topological black holes in dilaton gravity theory, Phys. Lett. B 612, 127 (2005).

[50] A. Sheykhi, M. H. Dehghani, and S. H. Hendi, Thermodynamic instability of charged dilaton black holes in AdS spaces, Phys. Rev. D 81, 084040 (2010).

[51] A. Sheykhi, S. H. Hendi, F. Naeimipour, S. Panahiyan, and B. E. Panah, Thermodynamic geometry of charged dilaton black holes in AdS spaces, Can. J. Phys. 94, 1045 (2016).

[52] S. J. Zhang and E. Abdalla, Holographic thermalization in charged dilaton anti-de Sitter spacetime, Nucl. Phys. B896, 569 (2015).

[53] A.c. Li, H. q. Shi, and D.f. Zeng, Phase structure and quasinormal modes of a charged AdS dilaton black hole, Phys. Rev. D 97, 026014 (2018).

[54] W. L. Xu and Y.C. Huang, Dynamics of domain wall in charged AdS dilaton black hole spacetime, Int. J. Mod. Phys. A 34, 1950132 (2019).

[55] H. J. Boonstra, K. Skenderis, and P. K. Townsend, The domain wall/QFT correspondence, J. High Energy Phys. 01 (1999) 003.

[56] K. Behrndt, E. Bergshoeff, R. Halbersma, and J. P. van der Schaar, On domain wall/QFT dualities in various dimensions, Classical Quantum Gravity 16, 3517 (1999).

[57] M. Cvetic, H. Lu, and C. N. Pope, Domain walls with localized gravity and domain wall/QFT correspondence, Phys. Rev. D 63, 086004 (2001).

[58] W. Israel, Singular hypersurfaces and thin shells in general relativity, Nuovo Cimento B 44, 1 (1966); Erratum, Nuovo Cimento B 48, 463 (1967).

[59] V. Balasubramanian and P. Kraus, A stress tensor for anti-de Sitter gravity, Commun. Math. Phys. 208, 413 (1999).

[60] Z. Dayyani, A. Sheykhi, and M. H. Dehghani, Counterterm method in Einstein dilaton gravity and the critical behavior of dilaton black holes with a power-Maxwell field, Phys. Rev. D 95, 084004 (2017).

[61] R. G. Cai and N. Ohta, Surface counterterms and boundary stress energy tensors for asymptotically nonAnti-de Sitter spaces, Phys. Rev. D 62, 024006 (2000).

[62] G. W. Gibbons and S. W. Hawking, Action integrals and partition functions in quantum gravity, Phys. Rev. D 15, 2752 (1977).

[63] S. de Haro, K. Skenderis, and S. N. Solodukhin, Gravity in warped compactifications and the holographic stress tensor, Classical Quantum Gravity 18, 3171 (2001).

[64] S. S. Gubser, AdS/CFT and gravity, Phys. Rev. D 63, 084017 (2001).

[65] L. Anchordoqui, C. Nunez, and K. Olsen, Quantum cosmology and AdS/CFT, J. High Energy Phys. 10 (2000) 050.

[66] E. P. Verlinde, On the holographic principle in a radiation dominated universe, arXiv:hep-th/0008140

[67] Y. Brihaye, C. Herdeiro, and E. Radu, Black hole spontaneous scalarisation with a positive cosmological constant, Phys. Lett. B 802, 135269 (2020).

[68] H. R. C. Ferreira and C. A. R. Herdeiro, Stationary scalar clouds around a BTZ black hole, Phys. Lett. B 773, 129 (2017).

[69] C. A. R. Herdeiro and E. Radu, Static Einstein-Maxwell black holes with no spatial isometries in AdS space, Phys. Rev. Lett. 117, 221102 (2016).

[70] D.f. Zeng, Exact inner metric and microscopic state of $\mathrm{AdS}_{3}$-Schwarzschld BHs, arXiv:1812.06777.

[71] D.f. Zeng, Schwarzschild fuzzball and explicitly unitary Hawking radiation, Nucl. Phys. B930, 533 (2018).

[72] D.f. Zeng, Resolving the Schwarzschild singularity in both classic and quantum gravity, Nucl. Phys. B917, 178 (2017).

[73] D. Glavan and C. Lin, Einstein-Gauss-Bonnet Gravity in 4-Dimensional Space-Time, Phys. Rev. Lett. 124, 081301 (2020). 